\title{
entos: A quantum molecular simulation package
}

Frederick R. Manby, ${ }^{1,}$ a) Thomas F. Miller III, ${ }^{2}$ b) Peter J. Bygrave, ${ }^{1}$ Feizhi Ding, ${ }^{2}$ Thomas Dresselhaus, ${ }^{1}$ Fidel A. Batista-Romero, ${ }^{1}$ Alexander Buccheri, ${ }^{1}$ Callum Bungey, ${ }^{1}$ Sebastian J. R. Lee, ${ }^{2}$ Rocco Meli, ${ }^{1,}$ c) Kaito Miyamoto, ${ }^{1,}$ d) Casper Steinmann, ${ }^{1,}$ e) Takashi Tsuchiya, ${ }^{1}$ Matthew Welborn, ${ }^{2}$ Timothy Wiles, ${ }^{1}$ and Zack Williams ${ }^{1}$

1) Centre for Computational Chemistry, School of Chemistry, University of Bristol, Bristol BS8 1TS, United Kingdom

${ }^{2)}$ Division of Chemistry and Chemical Engineering, California Institute of Technology, Pasadena, CA 91125

entos is designed for ab initio MD simulations of molecular and condensed-phase chemical reactions and other processes, with particular focus on mean-field and quantum embedding methods for electronic structure. The entos software package is developed in the $\mathrm{C}++14$ programming language with a structure that enables flexibility (by providing a long-term sustainable platform for development of methods in this area), efficiency (via task-based multi-threaded parallelism), and rigorous software engineering standards.

\section{SUMMARY}

entos is a software package that enables ab initio molecular dynamics calculations on molecular and condensed-phase chemical reactions and other processes. entos focuses on multiscale embedding methods that allow for accurate simulation of a small, chemically important region, in a larger, complex chemical environment. Key features include efficient implementations of densityfunctional theory (DFT) ${ }^{112}$ density-corrected $\mathrm{DFT}, \frac{[3}{1} \mathrm{em}$ bedded mean-field theory (EMFT), ${ }^{4} 7$ and Grimme's semi-empirical tight-binding method GFN-xTB ${ }^{8}$ For all of these methods, energy, gradient and semi-analytic Hessian calculations are available. For DFT and EMFT, excited-state calculations are possible through linear response and $\triangle \mathrm{SCF}$. All methods support calculations on fractional particle number and fractional spin systems. $\mathrm{QM} / \mathrm{MM}$ calculations are made possible via combination with external molecular dynamics packages. Classical and quantized molecular dynamics simulations (via ringpolymer molecular dynamics ${ }^{9}$ ) can be performed with any available energy method.

entos is developed in the $\mathrm{C}++14$ programming language with a clear and well-designed structure. The design aims included flexibility (in the sense of providing a long-term sustainable platform for development of methods in this area), efficiency (which is realized through a task-based parallelism model), and software engineering standards geared towards industrial use. Parallelism is achieved using the Intel Threaded Building Blocks library 10 The code has been developed using modern software engineering best practices, including version control, unit testing, test coverage analysis, and continuous integration.

Public release of entos is scheduled for calendar year 2019.

\footnotetext{
a) Electronic mail: fred.manby@bristol.ac.uk

b) Electronic mail: tfm@caltech.edu

c) Present address: Department of Biochemistry, University of Oxford, South Parks Road, Oxford OX1 3QU, United Kingdom

d) Present address: Toyota Central R\&D Labs., Inc., 41-1,
}

\section{ACKNOWLEDGEMENTS}

We gratefully acknowledge financial support from: the Engineering and Physical Sciences Research Council (EPSRC) (grants EP/M013111/1, EP/P022308/1, $\mathrm{EP} / \mathrm{R} 011656 / 1, \mathrm{EP} / \mathrm{R} 014493 / 1)$; the EPSRC Centre for Doctoral Training in Theory and Modeling in the Chemical Sciences (EP/L015722/1); the Royal Society Newton International Fellowship programme; the U.S. Department of Energy (DE-FOA-0001912); the Joint Center for Artificial Photosynthesis, a DOE Energy Innovation Hub, supported through the Office of Science of the U.S. Department of Energy under Award No. DE-SC0004993; the Caltech Resnick Sustainability Institute, The Dow Chemical Company, and Toyota Central R\&D Labs, Inc.

\section{REFERENCES}

${ }^{1}$ R. G. Parr and W. Yang, Density-functional theory of atoms and molecules (Oxford Univ. Press, 1989) pp. 1-197.

${ }^{2}$ T. C. Wiles and F. R. Manby, J. Chem. Theory Comput. 14, 4590 (2018)

${ }^{3}$ A. Wasserman, J. Nafziger, K. Jiang, M.-C. Kim, E. Sim, and K. Burke, Ann. Rev. Phys. Chem. 68, 555 (2017)

${ }^{4}$ M. E. Fornace, J. Lee, K. Miyamoto, F. R. Manby, and T. F. Miller III, J. Chem. Theory Comput. 11, 568 (2015).

${ }^{5}$ S. J. Lee, K. Miyamoto, F. Ding, F. R. Manby, and T. F. Miller III, Chem. Phys. Letts. 683, 375 (2017)

${ }^{6}$ F. Ding, F. R. Manby, and T. F. Miller III, J. Chem. Theory Comput. 13, 1605 (2017).

${ }^{7}$ F. Ding, T. Tsuchiya, F. R. Manby, and T. F. Miller III, J. Chem. Theory Comput. 13, 4216 (2017)

${ }^{8}$ S. Grimme, C. Bannwarth, and P. Shushkov, J. Chem. Theory Comput. 13, 1989 (2017)

${ }^{9}$ S. Habershon, D. E. Manolopoulos, T. E. Markland, and T. F. Miller III, Ann. Rev. Phys. Chem. 64, 387 (2013)

10 https://www.threadingbuildingblocks.org

Yokomichi, Nagakute, Aichi 480-1192, Japan

e) Department of Chemistry and Bioscience, Fredrik Bajers Vej 7H, 9220 Aalborg, Denmark 